\title{
The SUMER Ly- $\alpha$ line profile in quiescent prominences
}

\author{
W. $\operatorname{Curdt}^{1}$, H. Tian ${ }^{1,2}$, L. Teriaca ${ }^{1}$, and U. Schühle ${ }^{1}$ \\ 1 Max-Planck-Institut für Sonnensystemforschung, Max-Planck-Str. 2, 37191 Katlenburg-Lindau, Germany \\ e-mail: curdt@mps.mpg.de \\ 2 School of Earth and Space Sciences, Peking University, PR China
}

Received 15 December 2009 / Accepted 5 February 2010

ABSTRACT

\begin{abstract}
Aims. As the result of a novel observing technique, we publish for the first time $\mathrm{SoHO}$-SUMER observations of the true spectral line profile of hydrogen Lyman- $\alpha$ in quiescent prominences. Because $\mathrm{SoHO}$ is not in Earth orbit, our high-quality data set is free of geocoronal absorption. We studied the line profile to complement earlier observations of the higher Lyman lines and to substantiate recent model predictions.

Methods. We applied the reduced-aperture observing mode to two prominence targets and did a statistical analysis of the line profiles in both data sets. In particular, we investigated the shape of the profile, the radiance distribution, and the line shape-to-radiance interrelation. We also compared Ly- $\alpha$ data to co-temporal $\lambda 1206$ Si III data.

Results. We find that the average profile of Ly- $\alpha$ has a blue-peak dominance and is reversed more if the line-of-sight is perpendicular to the field lines. The contrast of Ly- $\alpha$ prominence emission rasters is very low, and the radiance distribution differs from the lognormal distribution of the disk. Features in the Si III line are not always co-spatial with Ly- $\alpha$ emission.

Conclusions. Our empirical results support recent multi-thread models, which predict that asymmetries and depths of the self-reversal depend on the orientation of the prominence axis relative to the line-of-sight.
\end{abstract}

Key words. Sun: UV radiation - Sun: filaments, prominences - line: formation - line: profiles - opacity

\section{Introduction}

Prominences protruding out of the perfect sphere of the visible solar disk are even visible with the naked eye, when the bright disk is occulted. These enigmatic features, which apparently withstand gravity, have attracted scientists for centuries, but despite substantial progress and effort during the past decades in understanding the physics of prominences, important aspects are still not understood. We refer to review articles and reference material that show this effort and are relevant to our work: e.g., Tandberg-Hanssen (1995); Patsourakos \& Vial (2002); Parenti et al. (2005); Wilhelm et al. (2007). Our own work focuses on prominence observations of the hydrogen Ly- $\alpha$ line profile, which reveal information on the physical conditions for the line formation.

The Ly- $\alpha$ line profile of all disk features is self-reversed (Curdt et al. 2001). Among other parameters the reversal is related to the amount of neutral hydrogen in its ground state, which by itself is a complex function of the temperature and density structure of the emitting plasma. In addition, flows of the emitting or the absorbing plasma and magnetic field may modulate the sizes of the red or the blue peak and the symmetry of the profile (Curdt et al. 2008; Tian et al. 2009a).

Early observations of the Ly- $\alpha$ line profile in prominences were completed with the LPSP instrument on OSO 8 (Vial 1982) and the UVSP instrument on SMM (Fontenla et al. 1988). These photoelectric measurements had to be corrected for the geocoronal absorption. They have already shown signatures of asymmetry and a wide parameter range for the depth of the reversal of the profile, features that at that time could not be reproduced by radiative transfer calculations. Later on, modeling made it clear that the overall emergent profile strongly depends on the physical conditions in the prominence (e.g., Gouttebroze et al. 1993). In particular, the imprint of the incident profile and the role of the prominence corona transition region (PCTR) were now employed to reproduce observations (e.g., Vial et al. 2007).

Recently, 2D-multithread models have been established, which are based on the theoretical work of Heinzel \& Anzer (2001) and predict - depending on the orientation of the prominence axis relative to the line of sight (LOS) - opposite asymmetries for the Ly- $\alpha$ and Ly- $\beta$ lines (Gunár et al. 2007, 2008) and deeply or less deeply self-reversed profiles (Schmieder et al. 2007). This is the dedicated context and the rationale of our work.

\section{Observations}

Because of its wavelength range from $660 \AA$ to $1600 \AA$, its high spectral resolution, and its vantage point outside of the irritating geocorona, which absorbs Ly- $\alpha$ emission, the SUMER instrument on SoHO (Wilhelm et al. 1995) is ideally suited to providing information about the line profile. Its enormous brightness, however, exceeds the capabilities of the SUMER detectors, and Ly- $\alpha$ can only be observed in small sections of $50 \mathrm{px}$ on both sides of the detectors beneath a 1:10 attenuating grid. Unfortunately, the attenuation also exerts a modulation onto the line profile, which makes it difficult to interpret this data. Attempts to observe Ly- $\alpha$ in quiet Sun locations on the unattenuated bare section of the photocathode had difficultiy calibrating the local gain depression. First results from prominence data acquired in April and May 2005 have been reported by Gunár et al. (2006), Vial et al. (2007), and Gunár et al. (2008). 

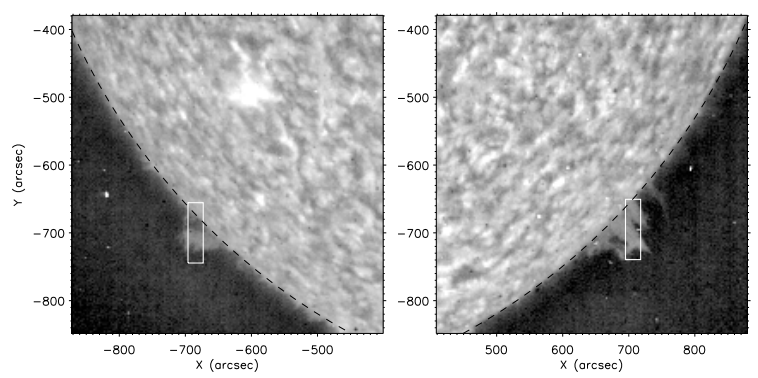

Fig. 1. The prominences observed above the southeast limb on June 15 (left) and above the southwest limb on June 9 (right). The images were taken in the EIT 304 channel (Delaboudinière et al. 1995), and the area of the SUMER rasters is indicated by rectangles.

In July 2008, the SUMER team found a new, unconventional method of observing the extremely bright Ly- $\alpha$ line of neutral hydrogen with partially closed telescope aperture to reduce the incoming photon flux. The obtained genuine Ly- $\alpha$ profiles in the quiet Sun and coronal hole regions were analyzed by Curdt et al. (2008), Tian et al. (2009a), and Tian et al. (2009b). Here we present the first unprecedented Ly- $\alpha$ observations of two quiescent prominences seen in June 2009 and discuss the results from a detailed analysis of the line profiles.

The new method reducing the incoming photon flux to a moderate level, appropriate for Ly- $\alpha$, was described in earlier work (Curdt et al. 2008; Tian et al. 2009a,b). A standard procedure for partially closing the door led to a reproducible reduction to a $20 \%$ level. In June 2009, this method was applied for the first time to prominence observations.

On June 9 and 15, 2009, we completed raster scans of approximately $22^{\prime \prime} \times 120^{\prime \prime}$ at positions near the solar limb with mid-size prominences. Two spectral windows were transmitted, 100 pixels (px) around Ly- $\alpha$ recorded on the bare photocathode of the detector and $50 \mathrm{px}$ around $\lambda 1206 \mathrm{Si}$ III recorded on the $\mathrm{KBr}$-coated section of the photocathode. All observations were completed with the $0.3^{\prime \prime} \times 120^{\prime \prime}$ slit. With an exposure time of $14.5 \mathrm{~s}$, both lines were observed with enough counts for a good line profile analysis. For both data sets three exposures at each position were completed before the raster was continued with a very small increment of $0.375^{\prime \prime}$. A first inspection indicated no temporal variations of the object during the observing time of $45 \mathrm{~min}$. In our statistical analysis we keep the temporal information and assume that the subresolution increment of $0.375^{\prime \prime}$ $\left(=3 / 8^{\prime \prime}\right)$ is equivalent to three hypothetical increments of $1 / 8^{\prime \prime}$.

Exact knowledge of the limb position and distance for each pixel is very important for prominence observations. Therefore we used additional information for an independent assessment of the pointing uncertainty, provided by the hardware encoders in the instrument's housekeeping channel. Thus, we confirmed that the azimuth movement was as expected and that the actual east-west pointing was very close to its nominal value. Similarly, we confirmed that in elevation the absolute positions for both rasters differ by the nominal value of $5^{\prime \prime}$. Since the position of the limb can be determined in the June 9 data set, we can estimate that the overall pointing uncertainty is about $2^{\prime \prime}$ to $3^{\prime \prime}$. The prominences as seen in the EIT 304 channel are shown in Fig. 1, including the area covered by the SUMER rasters.

Both data sets were processed with standard procedures of the SUMER-soft library. We used the dedicated flatfield exposure of April 19, 2009 to complete the flatfield correction.

\section{Prominences in Ly- $\alpha$ and in Si III}

The rasters for both days are shown in Figs. 2 and 3. The $x$-axis also contains time information (cf., prev. section), both axes are on a different scale, and the $x$-dimension is stretched. The contours delineate the top $15 \%$ and the top $40 \%$ of the pixels in the Ly- $\alpha$ brightness histogram. These contours have been transferred to the Si III raster.

In Fig. 2 (observation on June 15), we distinguish six different segments of the raster, separated by the blue contours or red boxes:

1. disk,

2. limb and near disk,

3. sub-prominence void,

4. inner prominence boundary,

5. prominence core,

6. outer prominence boundary.

We also display the averaged profiles of the designated areas. It is obvious that the disk profiles are much wider and more reversed than the prominence profiles. The disk profiles are almost symmetric, which is consistent with the downflow argument in Curdt et al. (2008) for this special geometry. Interestingly, a clear blue-peak dominance is observed in the profile of the prominence core.

In Fig. 3 (observation on June 9) we distinguish the

1. inner prominence core,

2. prominence interconnection,

3. sub-prominence void,

4. prominence interconnection,

5. outer prominence core,

6. outer prominence boundary.

For this, we observed just outside the limb. The contrast is even lower in this prominence, which is shown with the same dynamic range. The profiles are almost flat-topped, and significant reversals are not seen anywhere. The blue-peak dominance is also present in both parts of this prominence, but is less evident.

The prominence is also seen in Si III. Again, the Ly- $\alpha$ radiance contours have been transferred. These contours show that there are considerable differences in the Si III spectroheliogram, structures are not co-spatial, and the prominence appears more granulated and not as diffuse as in Ly- $\alpha$. The formation temperature of Si III is $70000 \mathrm{~K}$, much higher than typical prominence temperatures of $6000 \mathrm{~K}$ to $8000 \mathrm{~K}$. Si III is a typical transition region line. Since its wavelength is well above the Lyman-limit at $912 \AA$, opacity effects by hydrogen can be ruled out. The prominence is basically transparent (Anzer et al. 2007). These authors also show that the $\mathrm{C}$ I recombination continuum below $1239 \AA$ is negligible, and consequently the PCTR of each unresolved thread would contribute to the Si III emission so one would expect an appearance similar to the cold body. The differences in appearance may indicate the coexistence of hot and cold plasma with different opacities. Recent observations by Hinode-SOT (Berger et al. 2008) assume buoyant bubbles of hotter plasma in quiescent prominences, although on smaller scales. Such a scenario would also be compatible with our observation. Without Hinode-SOT co-observations, however, our results remain inconclusive.

We sorted the pixels of all disk locations and of all prominence locations by the total line radiance and defined six equally spaced radiance bins. The profiles for these bins are displayed in Figs. 4 and 5. There are striking differences of prominence profiles compared to disk profiles. In the prominence, the contrast is 

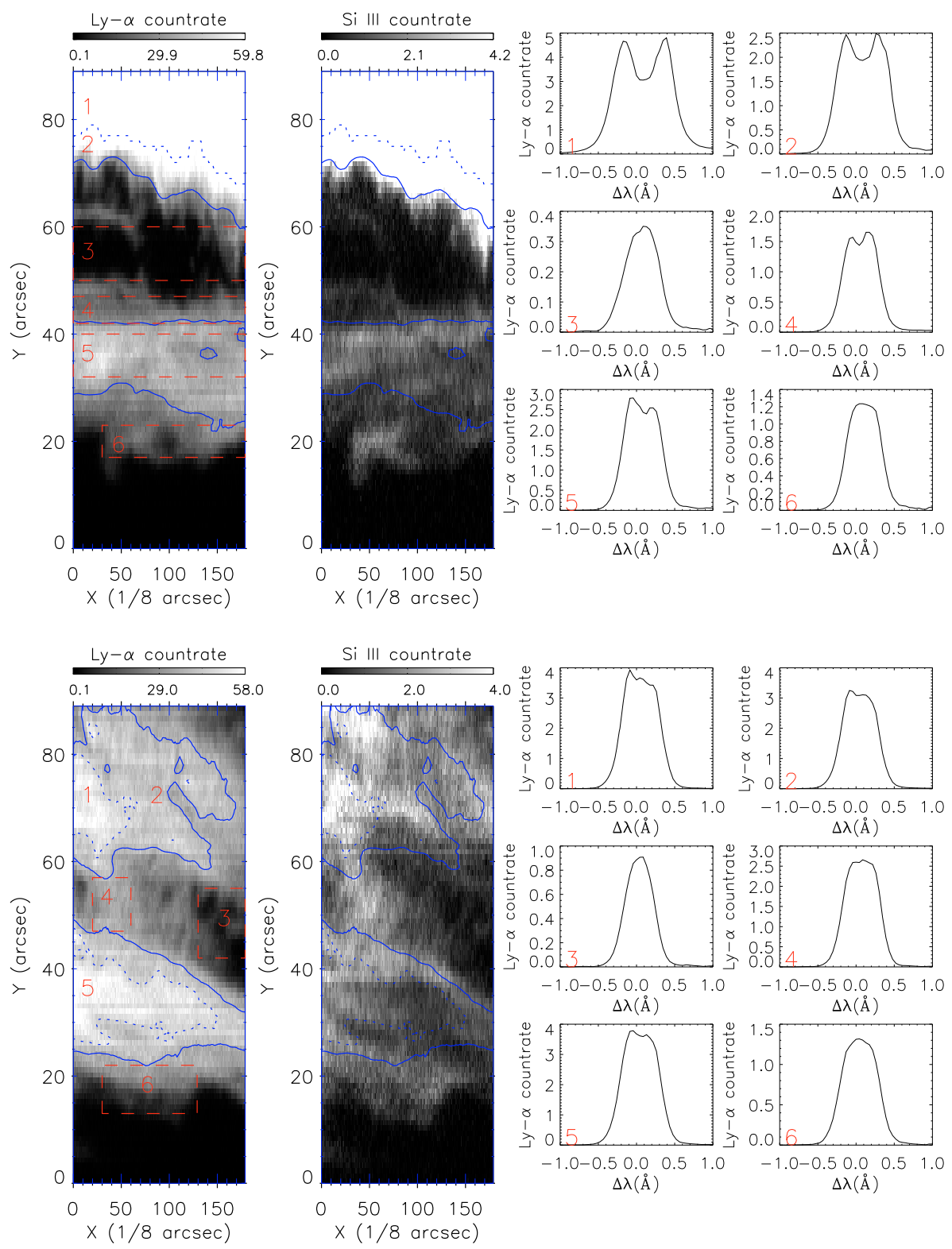

$\Delta \lambda(\AA)$

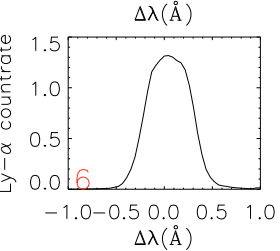

much lower, reduced by a factor of 4 to 5 . The blue-peak dominance is observed in all radiance bins from the brightest areas of the prominence core.

The central reversals of the Ly- $\alpha$ profiles in both prominences differ, the profiles obtained on June 15 were more reversed than those from June 9. This may be related to the different orientations of the prominence axes as derived from EIT 304 (cf., Figs. 2 and 3) and Kanzelhöhe H- $\alpha$ images. On June 15, the threads were rather perpendicular to the line-of-sight, while more edge-on (LOS parallel to the field lines) on June 9. This explanation would be consistent with the model calculations and predictions of Heinzel et al. (2005). Observational evidence for such a scenario based on spectra of the higher Lyman lines, Ly2 to Ly7, has been reported by Schmieder et al. (2007).

\section{Radiance histograms}

We already noted the low contrast of the prominences in Ly- $\alpha$. In Fig. 6 we show radiance histograms of the prominence core and of the on-disk locations in Fig. 2. For comparison we add the log-normal radiance distribution of Ly- $\alpha$ in the quiet Sun as
Fig. 2. Raster scan in Ly- $\alpha$ and Si III of the prominence observed on June 15 . The line radiance is given in counts/px/s. The raster also covers a small disk section near the southeast limb. Solid and dotted contours represent top $40 \%$ and top $15 \%$, respectively. The Ly- $\alpha$ contours have been transferred to the Si III raster. We show the average profiles of Ly- $\alpha$ of six distinguished locations (again in instrumental units; see text).

Fig. 3. Idem for the observation on June 9. The raster was completed just outside the southwest limb. We show the average profiles of Ly- $\alpha$ of six distinguished locations (see text).

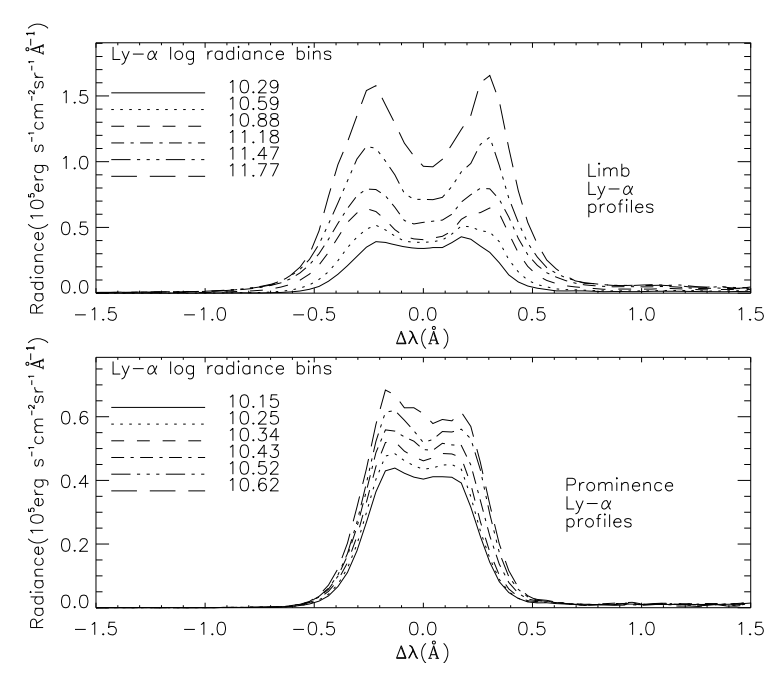

Fig. 4. We have sorted the pixels within the top $40 \%$ contours (solid in Fig. 2) by their radiance and show the profiles of Ly- $\alpha$ of six equally spaced radiance bins. The northern region represents disk and limb (top), the southern region the prominence core (bottom). 


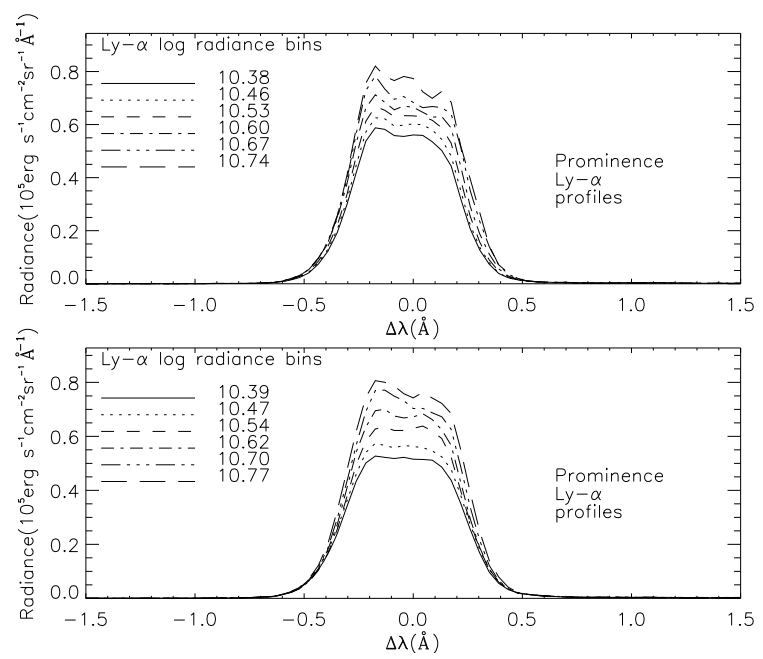

Fig. 5. Idem for the inner (top) and outer (bottom) region in Fig. 3.
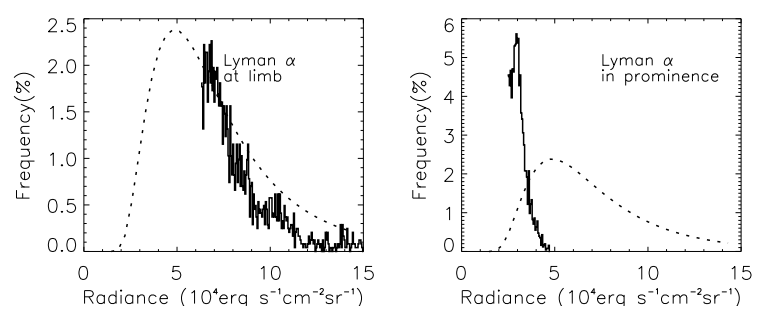

Fig. 6. Radiance distribution of the limb location $(1+2)$ in Fig. 2 (left) and of the prominence core (right). The low contrast of the prominence location translates into a narrow distribution, which differs significantly from the log-normal distribution, found by Curdt et al. (2008) in the quiet Sun at disk center (dotted curve).

presented in earlier work (Curdt et al. 2008). Because it has a different bin size, the disk histogram was scaled for better comparison. Although the small number of prominence pixels only allows a noisy distribution, the differences are, as expected, very obvious. The histogram is by definition clipped on the dim side because of the area selection criterion. The main difference is certainly found in the high-radiance part, because the prominence histogram completely lacks brighter pixels, which makes it a very narrow distribution. The uniform emergent emission translates, according to the Barbier-Eddington relation, to a uniform source function at an optical depth, $\tau$, of unity and is indicative of homogeneous populations of the $1 \mathrm{~s}$ and $2 \mathrm{p}$ levels, and thus rather homogeneous thermodynamic conditions.

In Fig. 7 we show the radiance distribution of the prominence in Fig. 3 in Ly- $\alpha$ and in Si III emission. This data set has more prominence pixels and also allows fainter pixels to be included here. We defined an empirically determined discrimination level to separate prominence emission from coronal background and defined the lower-15\% radiance category as coronal background, which does not belong to the prominence. The Ly- $\alpha$ histogram has a sharp upper limit and, in contrast to the disk histogram, a low-radiance wing. The Si III histogram of this prominence differs significantly, as one could expect, from both the quiet Sun state and from its Ly- $\alpha$ counter part.

We conclude that the radiance distributions of both prominences are, as a consequence of dissimilar physical conditions, remarkably different from the log-normal distribution of the average quiet Sun (Fontenla et al. 1988; Curdt et al. 2008).
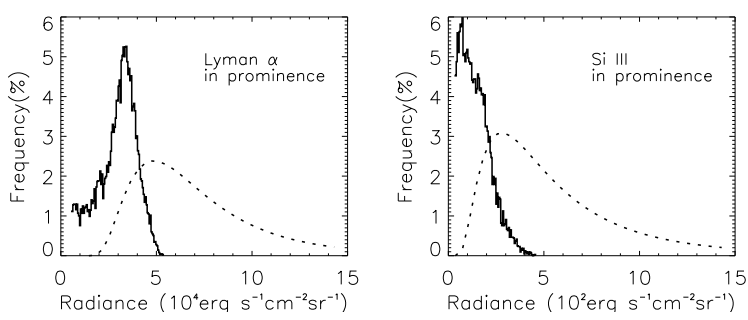

Fig. 7. Idem for the prominence in Fig. 3. The coronal background of pixels in the lower-15\% radiance category has been excluded.

\section{Summary and conclusion}

We have presented the first SUMER observations of prominences in the light of the hydrogen Ly- $\alpha$ line at $1216 \AA$ with reduced incoming photon flux to avoid the saturation effects of the SUMER detection system. We completed a statistical analysis and report salient empirical results derived thereof. As such, we found clear evidence of models, which predict an effect of the orientation of the magnetic field relative to the line of sight on the asymmetry of the Ly- $\alpha$ profile. The Lyman lines are more reversed if the line of sight is across the prominence axis as compared to the case where it is aligned along its axis. Given the great variability in the appearance of prominences and the wide range of physical parameters, the observation of two prominences is hardly enough to cover all the issues. We felt, however, that our results constitute a piece of information that is important enough to be presented here. More joint observations of prominences and modeling of their Ly- $\alpha$ line profile are highly desirable.

Acknowledgements. The SUMER project is financially supported by DLR, CNES, NASA, and the ESA PRODEX Programme (Swiss contribution). SUMER is part of SoHO of ESA and NASA. H.T. is supported by the International Max Planck Research School for his stay at MPS. This non-routine observation was performed with the help of D. Germerott. This paper greatly benefited from the very constructive comments of the referee.

\section{References}

Anzer, U., Heinzel, P., \& Fárník, F. 2007, Sol. Phys. 242, 42

Delaboudinière, J.-P., Artzner, G. E., Brunaud, J., et al. 1995, Sol. Phys. 162, 291 Curdt, W., Brekke, P., Feldman, U., et al. 2001, A\&A, 375, 591

Curdt, W., Tian, H., Teriaca, L., Schühle, U., \& Lemaire, P. 2008, A\&A, 492, L9

Berger, T. E., Shine, R. A., Slater, G. L., et al. 2008, ApJ, 676, L89

Fontenla, J. M., Reichmann, E. J., \& Tandberg-Hanssen, E. 1988, ApJ, 329, 464 Gunár, S., Teriaca, L., Heinzel, P., \& Schühle, U. 2006, in Proc. SoHO-17. 10

Years of SoHO and Beyond, ed. H. Lacoste, \& L. Ouwehand, Giardini Naxos, Italy, ESA SP-617, 63

Gunár, S., Heinzel, P., Schmieder, B., Schwartz, P., \& Anzer, U. 2007, A\&A, 472,929

Gunár, S., Heinzel, P., Anzer, U., \& Schmieder, B. 2008, A\&A 490, 307

Gouttebroze, Heinzel, P., \& Vial, J.-C. 1993, A\&AS, 99, 513

Heinzel, P., \& Anzer, U. 2001, A\&A, 375, 1082

Heinzel, P., Gouttebroze, P., Vial, J.-C., et al. 1987, A\&A, 183, 351

Heinzel, P., Anzer, U., \& Gunár, S. 2005, A\&A, 442, 331

Schmieder, B., Gunár, S., Heinzel, P., \& Anzer, U. 2007, Sol. Phys., 241, 53

Parenti, S., Vial, J.-C., \& Lemaire, P. 2005, A\&A, 443, 679

Patsourakos, S., \& Vial, J.-C. 2002, Sol. Phys., 208, 253

Tandberg-Hanssen, E. 1995, The Nature of Solar Prominences (Dordrecht: Kluwer), Ap\&SS Lib., 199

Tian, H., Curdt, W., Marsch, E., \& Schühle, U. 2009a, A\&A, 504, 239

Tian, H., Teriaca, L., Curdt, W., \& Vial, J.-C. 2009b, ApJ, 703, L152

Vial, J.-C. 1982, ApJ, 253, 330

Vial, J.-C., Ebadi, H., \& Ajabshirizadeh, A. 2007, Sol. Phys., 246, 327

Wilhelm, K., Curdt, W., Marsch, E., et al. 1995, Sol. Phys., 162, 189

Wilhelm, K., Marsch, E., Dwivedi, B. N., \& Feldman, U. 2007, SSRv, 133, 103 